\title{
GPR detection of buried steel gas cylinder; a study conducted in a sandbox
}

\author{
Van-Dycke Sarpong Asare \\ Department of Physics, Kwame Nkrumah University of Science and Technology, Kumasi \\ *Corresponding authorE-mail: vandyckea@yahoo.co.uk
}

\begin{abstract}
In this study a controlled medium was created to apply the ground penetrating radar technique to detect and locate a buried steel gas cylinder. The work was to demonstrate the utility of the GPR in locating underground utilities prior to excavation for civil works. The survey was carried out in a sandbox measuring $4.20 \mathrm{~m}$ by $2.30 \mathrm{~m}$ of surface cross section and a depth of $1.10 \mathrm{~m}$. The wooden box with an open bottom was filled with dry sand which had been sieved to rid it of organic matter and therefore to create a substantially homogenous medium. An empty LPG cylinder was buried in the sand at a measured location and depth from the sand surface. The measurement was carried out with the $800 \mathrm{MHz}$ shielded GPR antennae. The experiment led to a definition of the GPR signature of the horizontally oriented empty steel cylinder. The burial depth, representing the top surface of the cylinder from the sand surface was found to be in the range of 0.58 to $0.64 \mathrm{~m}$, compared with a concealed depth of $0.60 \mathrm{~m}$. The bottom of the sandbox was also well imaged and located within the range of $0.95 \mathrm{~m}$ to $1.20 \mathrm{~m}$. The actual depth of the sand was $1.10 \mathrm{~m}$.
\end{abstract}

Keywords: Dielectric Permittivity, Ground Penetrating Radar, Monostatic,, Scatterer, Radagrams.

\section{Introduction}

Ground penetrating radar (GPR) is a geophysical method that uses short radar pulses to image the subsurface. This method uses electromagnetic radiation in the microwave band (UHF/VHF frequencies) of the radio spectrum, and detects the reflected signals from subsurface structures. The reflections are due to the contrast in dielectric permittivity between the medium through which the wave is passing and that of the encountered material or object. GPR can be used in a variety of media, including rock, soil, ice, fresh water, pavements and structures. It can detect objects, changes in material, and voids and cracks [1].

Despite its relatively low penetration depth (especially with high-frequency antennae and in moderately conductive environments), the GPR resolution capability (also depending on frequency and soil properties), is by far greater than that obtained by other geophysical methods. This makes this technique suitable for high-resolution shallow studies like archaeological applications and shallow stratigraphy mapping [2].

This work demonstrates the utility of the GPR technique in locating underground utilities whose orientations are known, prior to excavation. These underground mains may include lines for telephones, electricity distribution, natural gas, cable television, fiber optics, traffic lights, street lights, storm drains, water mains, and wastewater pipes. In some locations, major oil and gas pipelines, national defense communication lines, rail and road tunnels also compete for space underground. For new constructions or repair works it is very important to locate existing underground utilities. If the utilities are of older age, the existing documentation is often non-existing or poor, which implies that accurate location becomes imperative.

The GPR technique is similar in principle to seismic; one transmitter antenna radiates short pulses of high-frequency $(\mathrm{MHz}$ to $\mathrm{GHz}$ ) electromagnetic waves, and the receiver antenna, then measures the arrival times of the reflected. The speed of the EM waves is related to the dielectric permittivity of the subsurface materials [3], [4]. For a lossless, non

$$
v=\frac{c}{\sqrt{\varepsilon_{m}}}
$$


magnetic medium, the velocity of EM waves is given in terms of the dielectric permittivity as, where c is the EM waves speed in free space. The propagation of radar signal in ground therefore depends on the dielectric permittivity of the soil. The dielectric permittivity of a material is a frequency-dependent response of the material to electromagnetic waves [5] and it can be used to distinguish it from other materials. Velocity information with respect to a particular subsurface structure can therefore be used to detect variation or discontinuity within the media of different dielectric property.

In GPR survey, the depth penetration is primarily a function of the antennae frequency. The penetration is however mitigated by the conductivity of the soils. Conductive soils such as clays attenuate radar signals much more rapidly than resistive soils, such as dry sand [6].

Underground utilities, like sewage pipes and electric cables are usually identified as hyperbolae if they are traversed perpendicular to their long axes. The hyperbolae represent the locus of the two-way travel time (TWT) as the transmitter-receiver of a monostatic system approaches, crosses and recedes the underground utility.

In a 2-D coordinate system is a point scatterer (the top surface of the cylinder) at a position $\left(0, z_{o}\right)$ in the half space, will be located by the antenna pair, and situated in $(\mathrm{x}, 0)$ at a distance $\left(x^{2}+z^{2}\right)$. The recorded data is represented by the two-way travel time of the reflection from the point scatterer. If the propagation velocity in the homogeneous half space is $\mathrm{v}$, appears at the receiver after a time

$$
t=2 \frac{\sqrt{x^{2}+z^{2}}}{v}
$$

Equation (1) represents a hyperbola with a vertical axis and an apex in $\left(0,2 z_{o} / v\right)$. Thus the shape of the hyperbola is function of the antenna configuration (monostatic in this case), the depth of the point scatterer, and the propagation velocity profile of the half space.

It has to be noted that occasionally the utility itself may not be seen, for instance when the dielectric properties of the utility and the surrounding media are identical. In such cases, sometimes a structure change in the radagram indicating dug or non-dug areas can be observed. In this way the ditch of the utility can be identified, and by that an approximate location of the utility [7].

\section{Method}

\subsection{Medium-The context}

For the success of this experiment, a wooden rectangular box of surface dimensions $4.20 \mathrm{~m}$ by $2.30 \mathrm{~m}$ and height 1.10 $\mathrm{m}$ was erected on a smooth flat ground surface. The structure was then filled with sieved but uncalibrated dry sand (Fig. 1) and the empty steel LPG cylinder buried inside the sand (Fig. 2). This ground condition-the homogeneous sand, was ideal for the GPR measurements because almost no other hyperbolic reflections would be seen in addition to that of the cylinder.

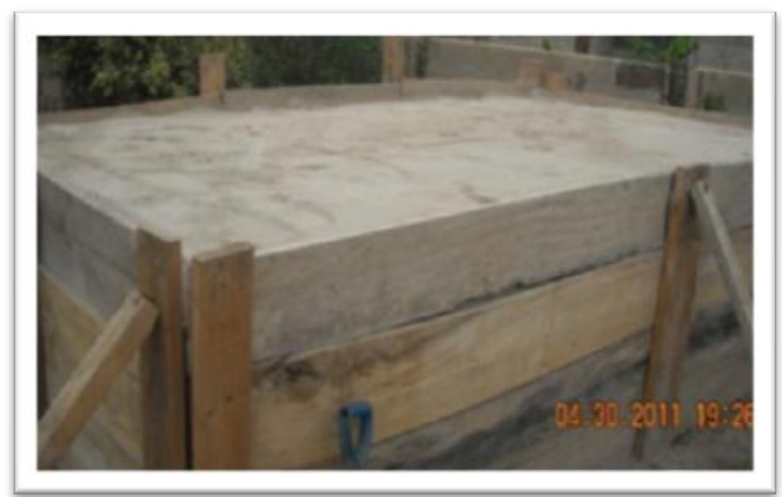

Fig. 1: Sandbox constructed on a level ground and filled with sieved sand

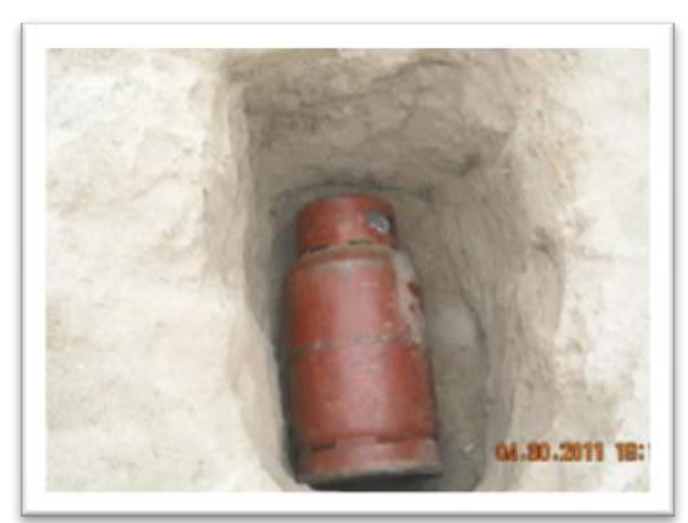

Fig. 2: A Steel cylinder buried in the sandbox 


\subsection{GPR measurements}

In this study the GPR measurements were conducted with the shielded $800 \mathrm{MHz}$ antennae of the RAMAC/GPR system. The centre of the cylinder is positioned at $P(x, y, z)$, where $x$ and $y$ are the respective coordinates along the length and breadth of sandbox and $z$ is the depth from the sand surface to the top of the cylinder (Fig. 3). In this study, the cylinder has only one axis of polarization - the long axis of the cylinder being perpendicular to the length of the sand box. Survey was conducted along profiles spaced $40 \mathrm{~cm}$ from each other at sampling space interval of $2 \mathrm{~cm}$ set on the acquisition wheel. 5 parallel monosatatic profiles of length $3.20 \mathrm{~m}$ were run along the long axis of the box, (Fig. 4). In this experimental study, the direction of the utility is known. That is why the survey lines were arranged perpendicular to the direction of the utility. Other acquisition parameters included, an $8000 \mathrm{MHz}$ sampling frequency, 500 samples per scan, 512 stacks for each trace and a time window of $22 \mathrm{~ns}$.

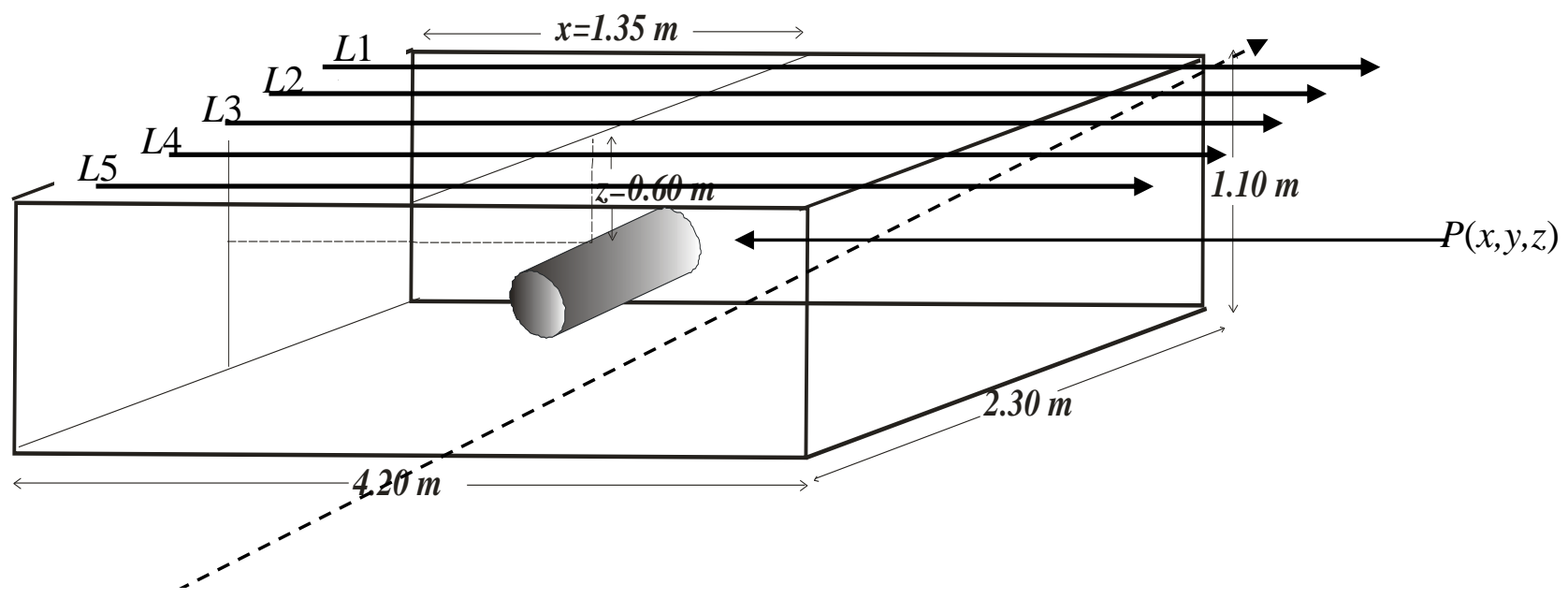

Axis of cylinder

Fig. 3: Plan view of the sandbox with the buried cylinder

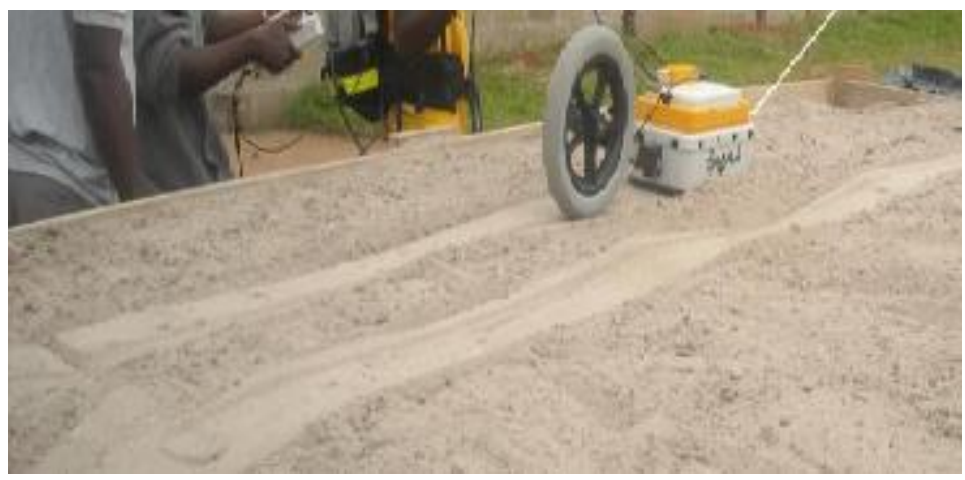

Fig. 4: The $800 \mathrm{MHz}$ antennae being pulled on the surface of the sand

\subsection{GPR data Processing}

The quality of the data was improved by removing the low frequency component (known as the DC component or dewow) with a running average filter in time. It is important to note that during the processing a linear gain control, and not automatic gain control (AGC) was applied. A linear gain control allows for the detection of small changes in signal amplitude which may be obliterated with the use of an AGC filter [8]. The same parameters were used for all data in order to keep the relative amplitudes of the signals from one data set to the other. These processing transactions are routines in the REFLEXW software, version 5 [9]. Other processing steps applied to the acquired radagrams were: static and dynamics corrections of the signals. The static corrections were done using positive polarization, which shows the 
period extended between the beginning of the first positive reflection and its end filter limit. The range in the time window was identified between zero and $22 \mathrm{~ns}$. Bandpass filtering was also applied. 2D filter process using background removal filter removed noise and artefactual horizontal layers within the time window extending from $2.0 \mathrm{~ns}$ to the 22.0 ns. Figs. 5a and $5 \mathrm{~b}$ respectively show examples of raw and processed radagrams.

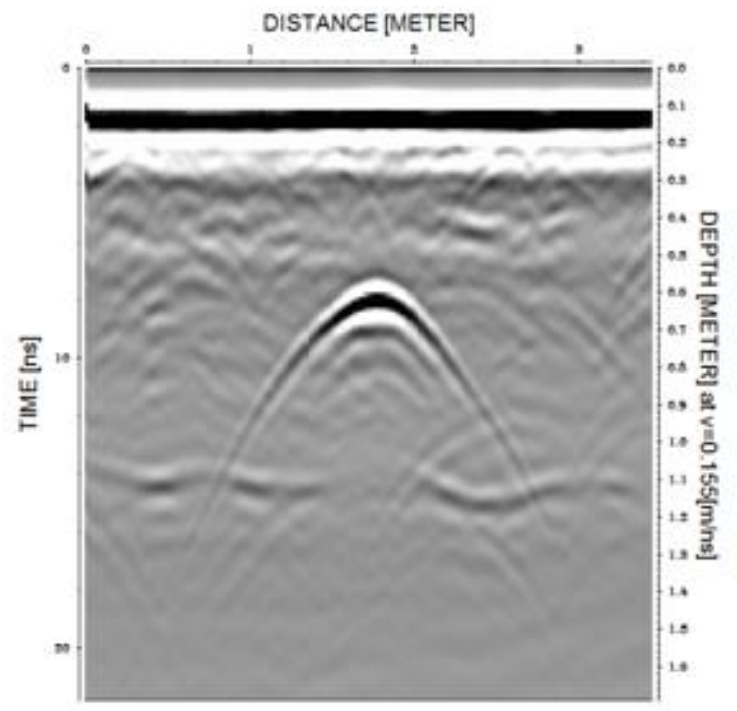

(a)

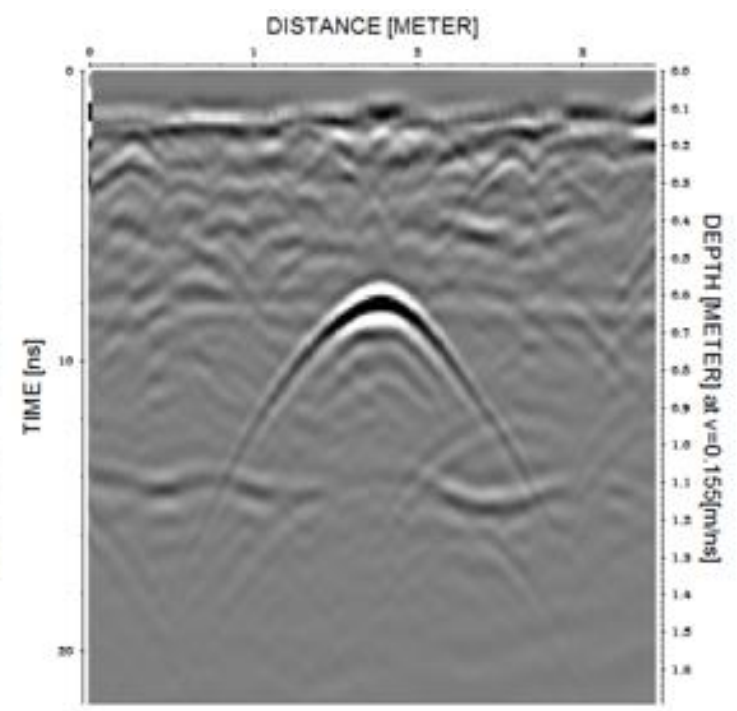

(b)

Fig. 5: (a) Raw and (b) Processed radagrams

\section{Results}

\subsection{GPR Sections}

The raw and processed GPR sections (radagrams) obtained along the five profiles have been produced in Figures 6 to 10 below. The sixth, Figure 11, is the section obtained when the cylinder was not present in the sand. The apex of the hyperbola represents the top surface of the cylinder. The profile distance, which represents the position of the top of the cylinder along the length of the box and the depth of burial of the cylinder are shown in each radagram.

\subsection{Velocity Analysis/Hyperbola Fitting}

In determining the depth of burial of the cylinder and the sand-ground interface, velocity analyses were performed in the REFLEX W software. The standard hyperbola fitting in the Reflex was applied to identify and characterize the buried cylinder. When the profile passes over the cylinder in the ground, the reflection from it is characterized by a hyperbola in the subsurface radar image. The shape of the hyperbola defined by equation (1) in the $x-t$ plane, is geometry and transmission velocity dependent.

The hyperbola fitting procedure is accomplished by interactively superposing a theoretical hyperbola that is congruent with the hyperbola in the radagram. The attributes of the theoretical hyperbola are assigned to the hyperbola in the radagram.

The profiles used for the hyperbola fitting, and indeed all the profile run in this experiment, are all perpendicular to the axis of the obstacle and therefore polarization effects do not arise. That is, the radar section is perpendicular to the longitudinal axis of the buried cylinder.

The gas cylinder used for the work has a cross-sectional radius of $0.16 \mathrm{~m}$. It should be noted that the hyperbola characteristics change as the diameter of the buried pipe increases. With this radius value of $0.16 \mathrm{~m}$ and an electromagnetic wave velocity of $0.140 \mathrm{~m} / \mathrm{hs}$, the two hyperbolas were congruous in all the profiles. For each, the profile distance, the depth to the top of the cylinder and the two-way travel time were obtained. These parameters put the depth of burial within the range of $0.58 \mathrm{~m}$ to $0.64 \mathrm{~m}$. 


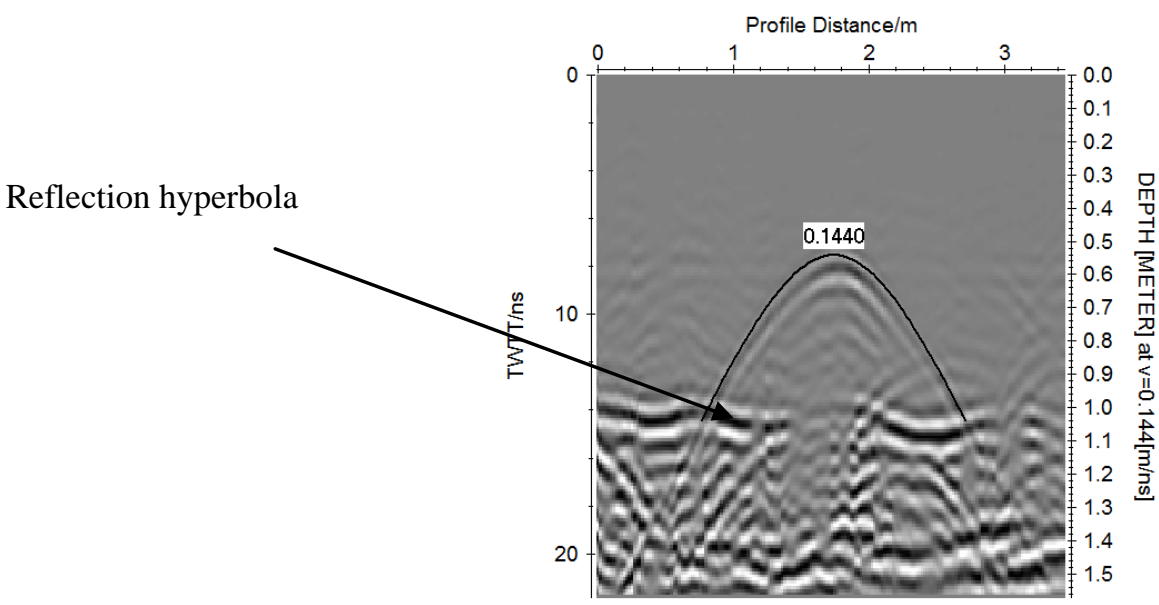

(i)
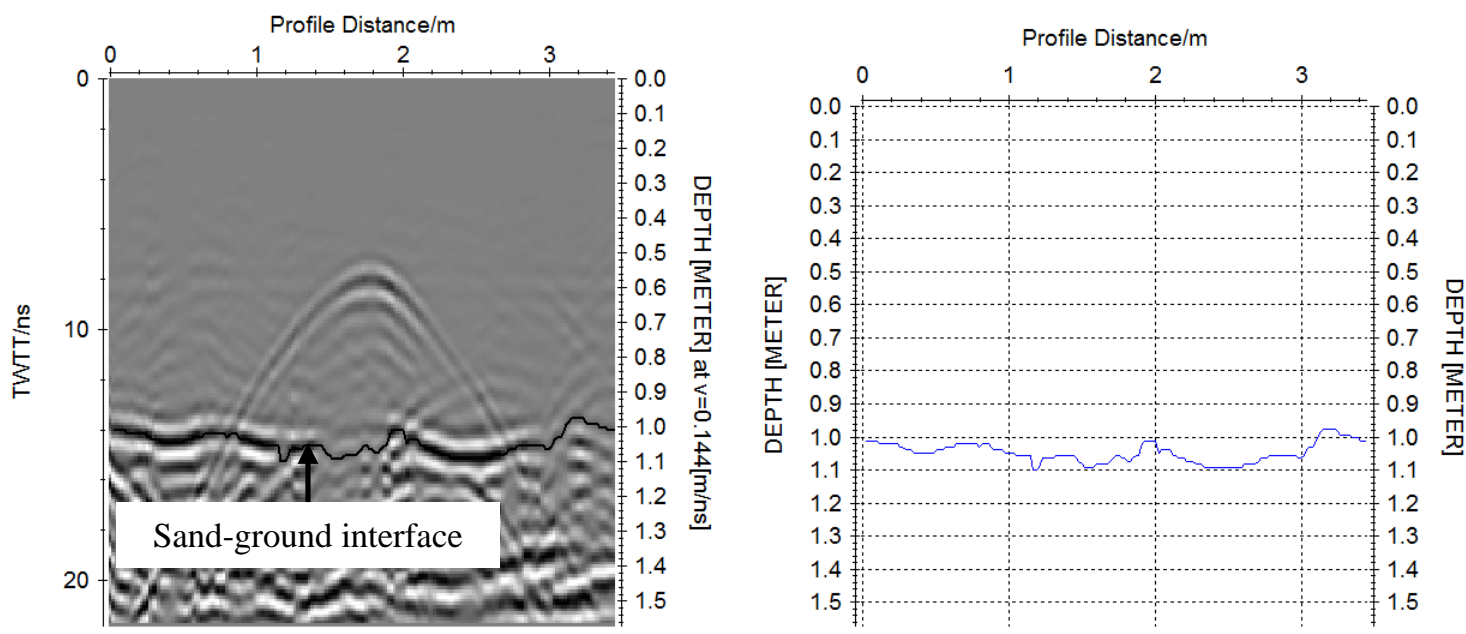

(ii)

Fig. 6: (i) Processed radagram of L1 showing the reflection hyperbola from the cylinder and (ii) Reflection horizon from the sand-ground interface and its interpreted section.

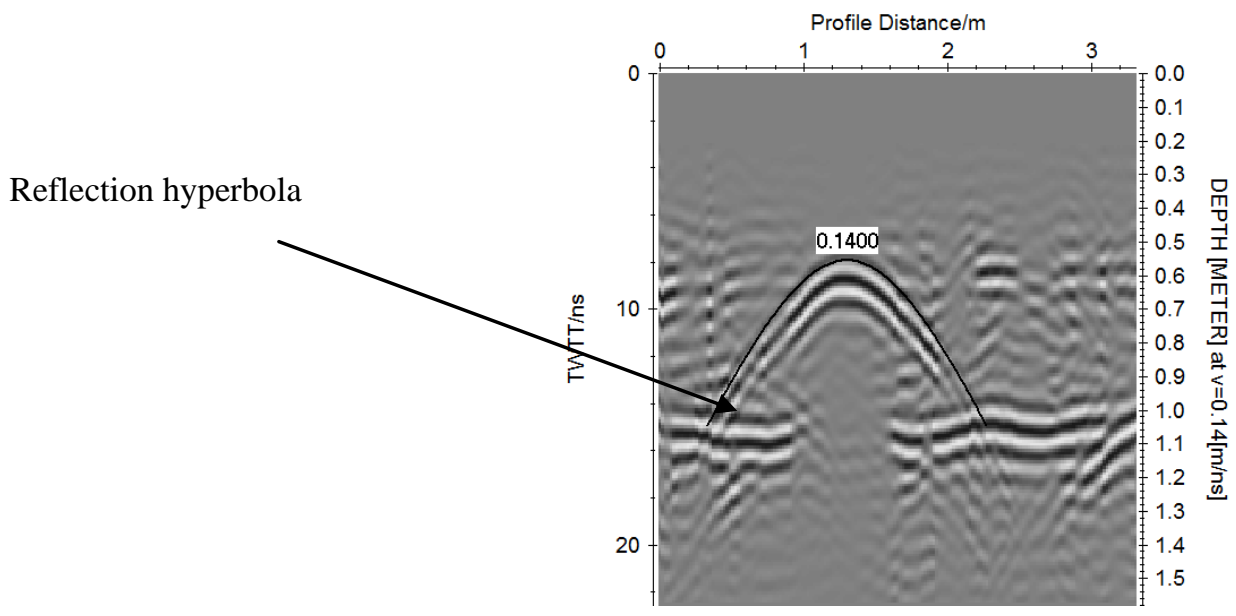

(i) 

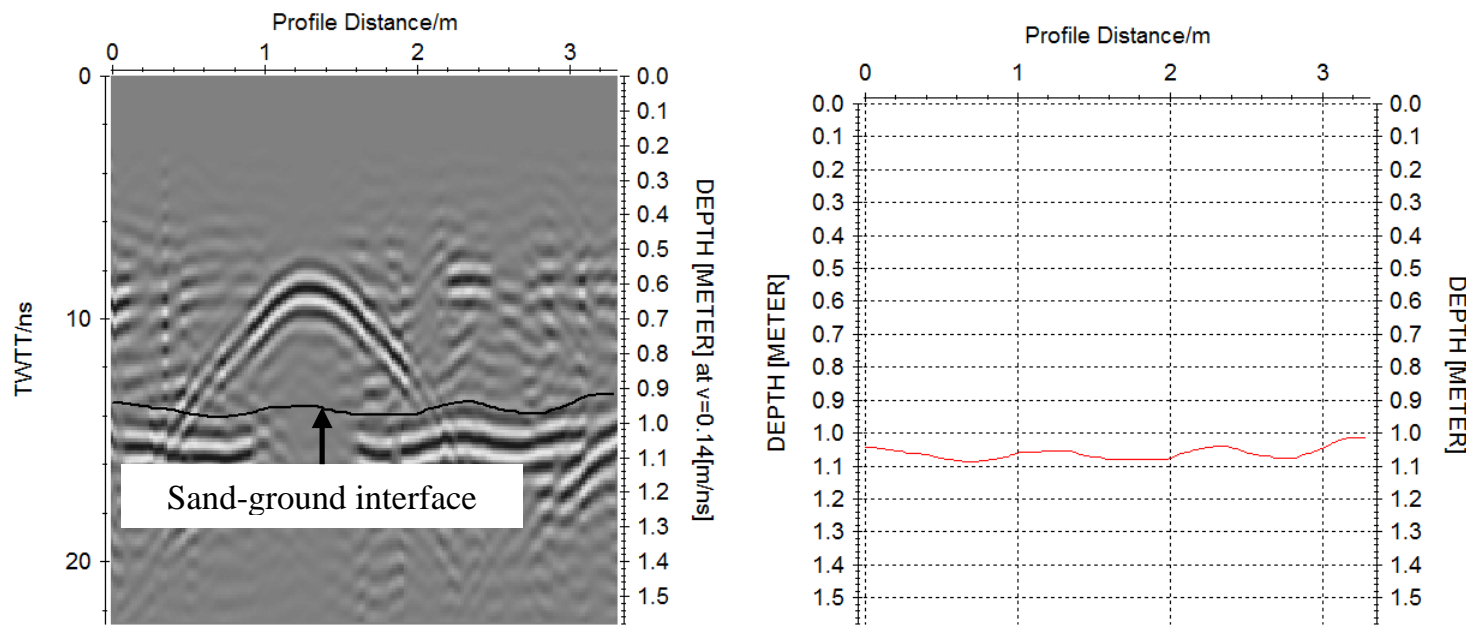

(ii)

Fig. 7: (i) Processed radagram of $\mathrm{L} 2$ showing the reflection hyperbola from the cylinder and (ii) Reflection horizon from the sand-ground interface and its interpreted section.

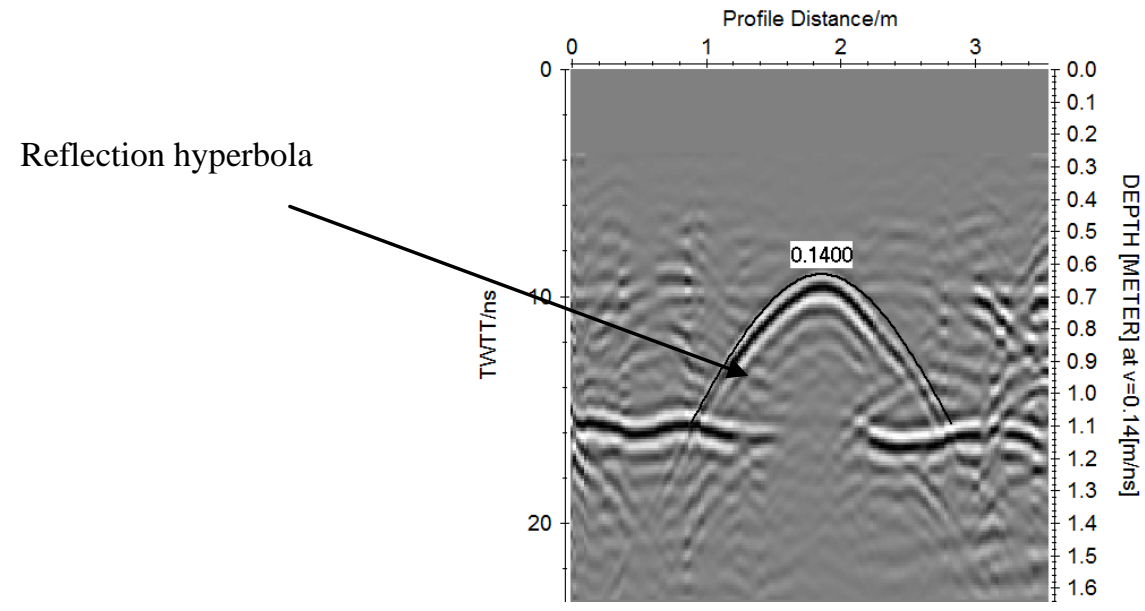

(i)
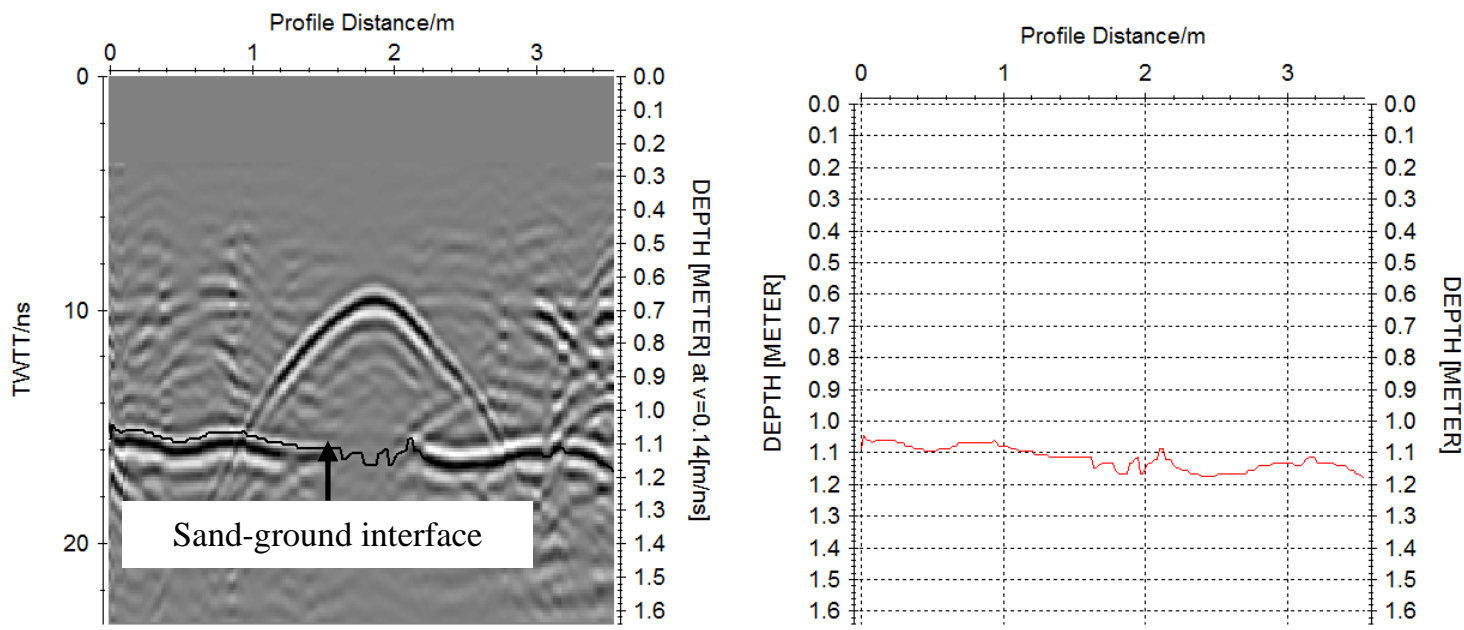

(ii)

Fig. 8: (i) Processed radagram of L3 showing the reflection hyperbola from the cylinder and (ii) Reflection horizon from the sand-ground interface and its interpreted section. 

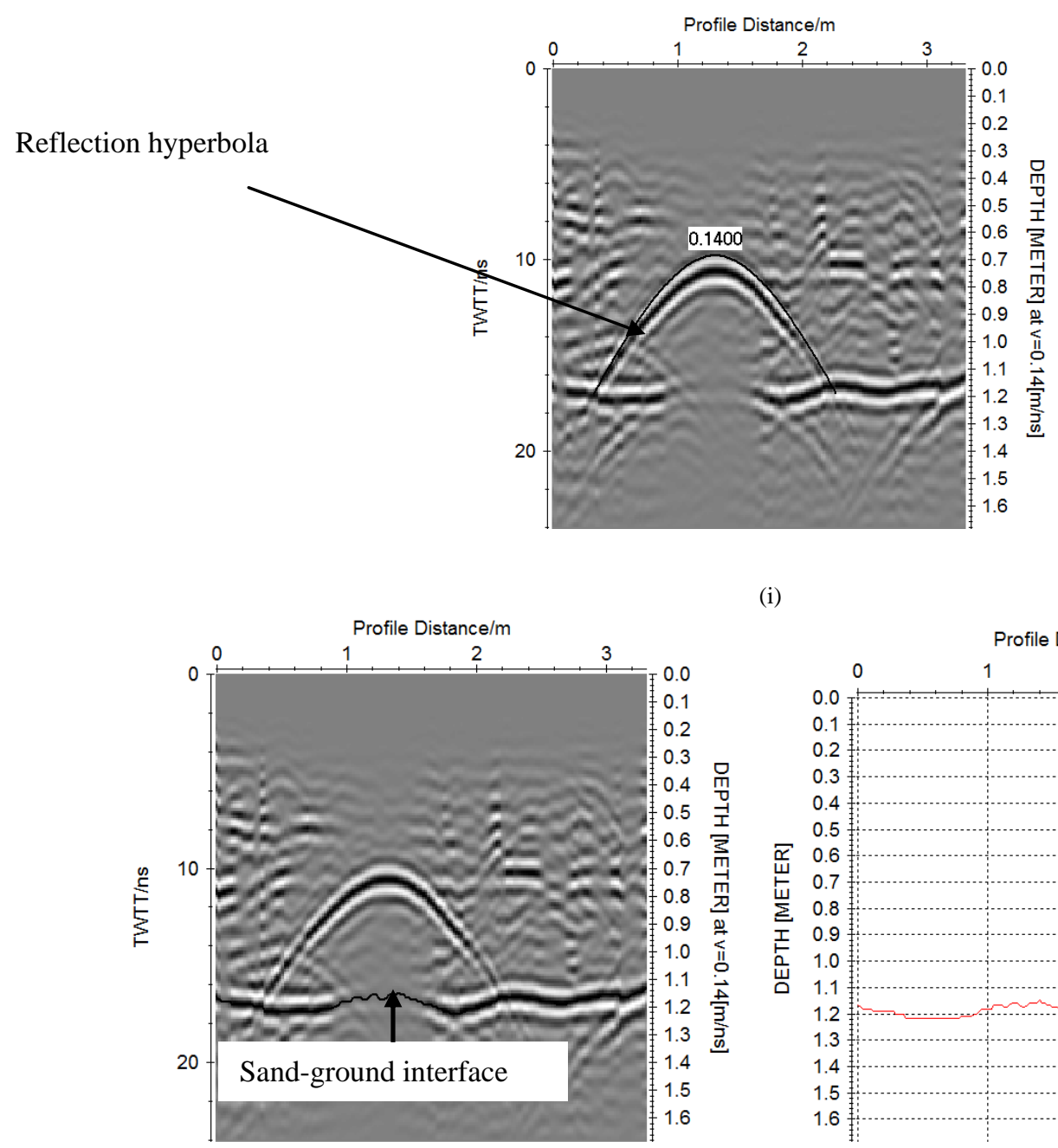

(i)

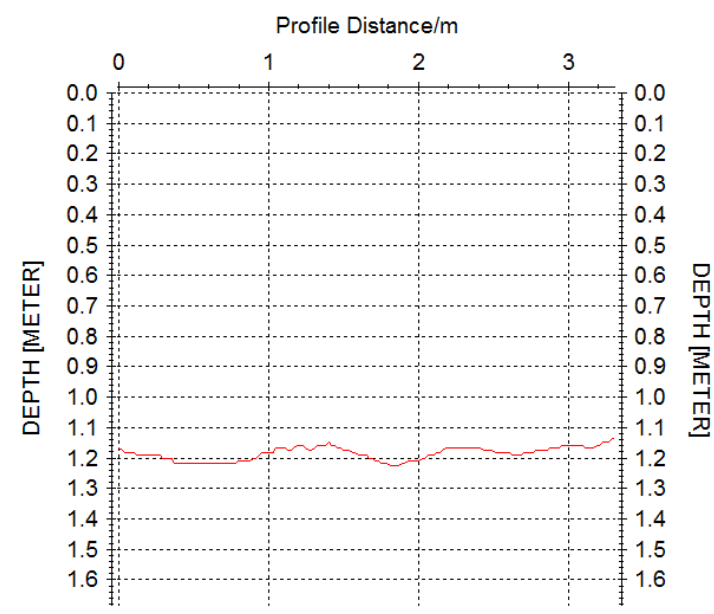

(ii)

Fig. 9. (i) Processed radagram of L4 showing the reflection hyperbola from the cylinder and (ii) Reflection horizon from the sand-ground interface and its interpreted section.

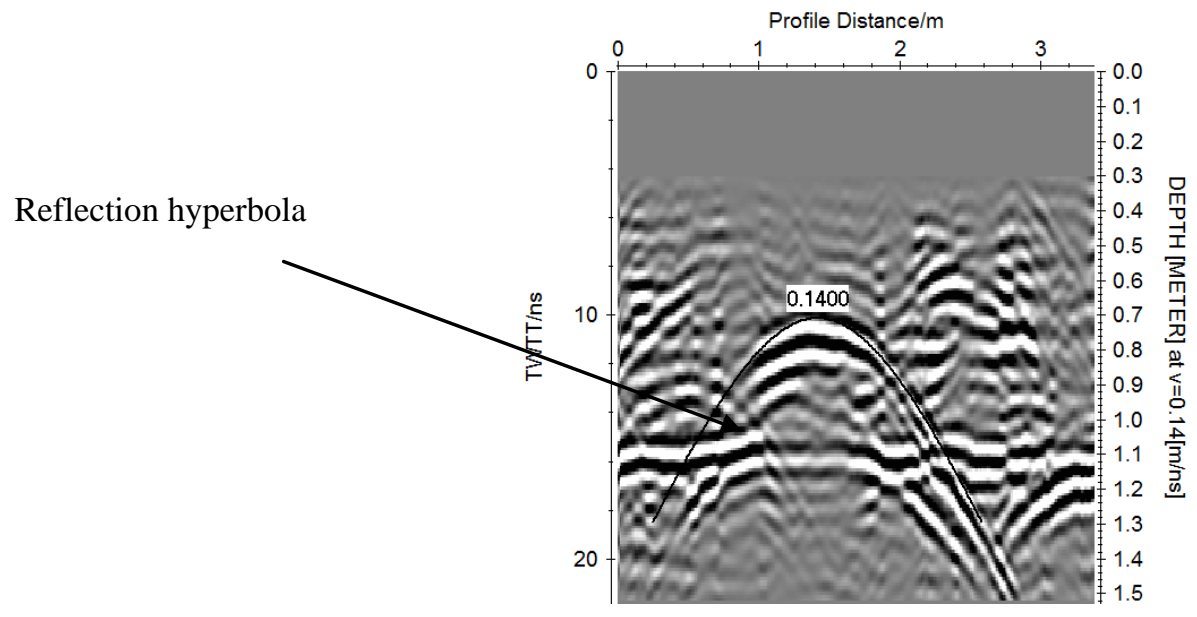

(i) 


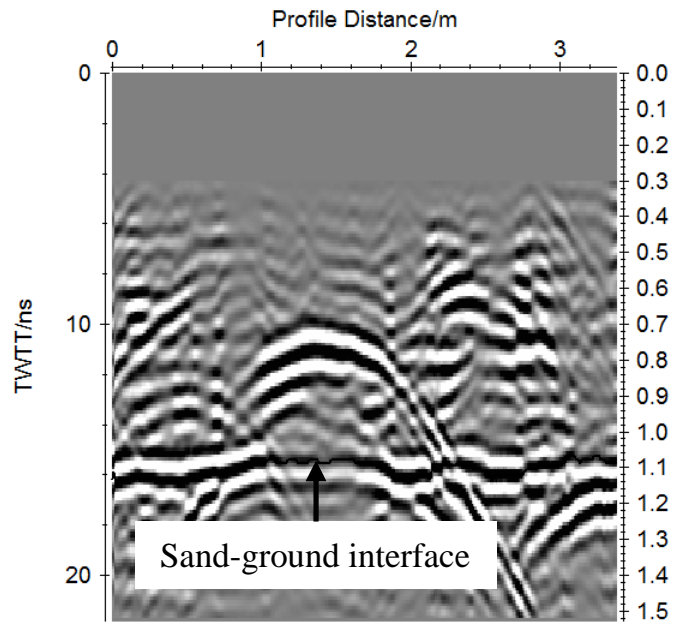

(ii)

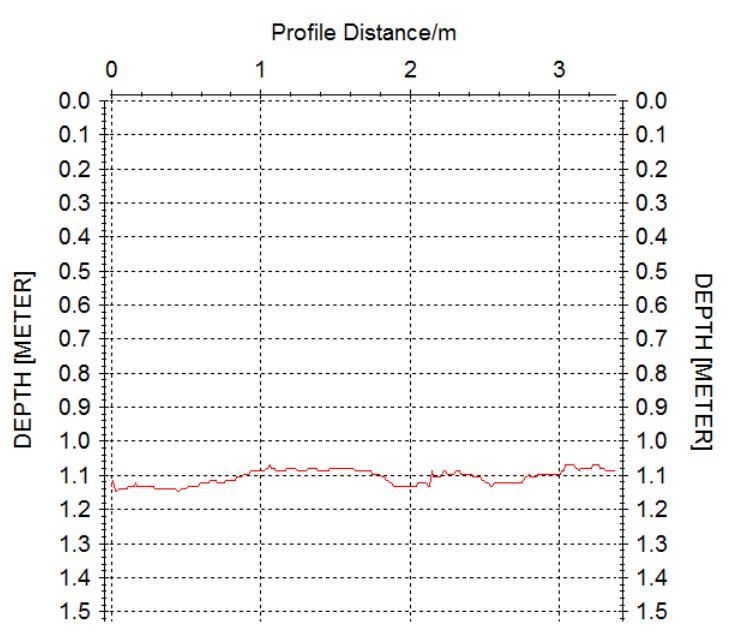

Fig. 10: (i) Processed radagram of L5 showing the reflection hyperbola from the cylinder and (ii) Reflection horizon from the sand-ground interface and its interpreted section.
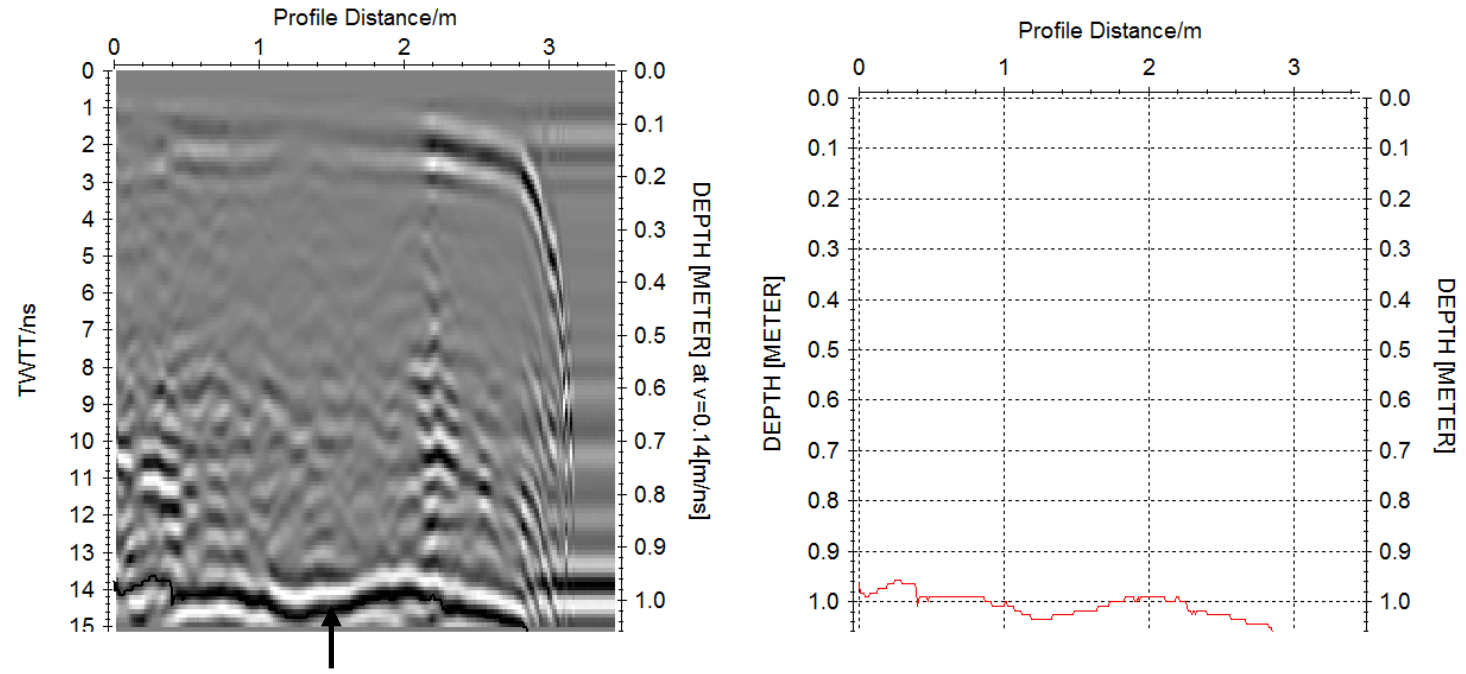

Sand-ground interface

Fig.11. Processed radagram showing reflection horizon from the sand-ground interface and its interpreted section of one of the lines conducted without the cylinder.

Table 1 below produces a summary of the results of the profiling. The specific results depicted in the table are the profile distances, the depths to the top of the cylinder and the respective two-way-travel times. For each profile the sand-ground interface depths are also provided in the Table. The sixth profile was conducted with the gas cylinder absent from the sandbox.

Table 1: Profile parameters

\begin{tabular}{|c|c|c|c|c|}
\hline \multirow{2}{*}{ Profile No } & \multicolumn{3}{|c|}{ Apex of hyperbola } & $\begin{array}{c}\text { Range of sand- } \\
\text { ground Interface } \\
\text { depth /m }\end{array}$ \\
\cline { 2 - 5 } & $\begin{array}{c}\text { Profile Distance } \\
\text { object/m }\end{array}$ & Depth of object/m & $\begin{array}{c}\text { Two-way travel } \\
\text { time/ns }\end{array}$ & 8.26 \\
\hline$(456) 1$ & 1.38 & 0.64 & 7.45 & $1.00-1.10$ \\
\hline$(451) 2$ & 1.77 & 0.58 & 7.35 & $0.95-1.00$ \\
\hline$(452) 3$ & 1.30 & 0.58 & 7.43 & $1.04-1.15$ \\
\hline$(453) 4$ & 1.85 & 0.58 & 7.72 & $1.08-1.20$ \\
\hline$(454) 5$ & 1.31 & 0.60 & -- & $0.98-1.05$ \\
\hline$(456) 6$ & -- & -- & & \\
\hline
\end{tabular}




\section{Discussions}

The radar image is migrated for a range of velocities in order to determine the velocity at which the characteristic hyperbola best focuses to a point - indicating the propagation velocity in the ground. Iteratively, an electromagnetic wave velocity of $0.140 \mathrm{~m} / \mathrm{ns}$ was found appropriate in defining the depth of burial of the cylinder and also the sandground interface. This velocity translates into a dielectric constant of 3.75 for sand. Dry sand has a dielectric constant in the range of 3.0 to 5.0 [10]. Using the $800 \mathrm{MHz}$ antenna, the depth to the top of the buried cylinder was found within the range of $0.58 \mathrm{~m}$ to $0.64 \mathrm{~m}$ in relation to the nominal concealed depth of $0.62 \mathrm{~m}$. The thick lines in the various processed GPR sections indicate the location of the sand-ground interface, which is substantially flat. The bottom of the sandbox is thus well imaged (thick line) and located within average depth range of $0.95 \mathrm{~m}$ to $1.20 \mathrm{~m}$. The actual thickness of sand was $1.10 \mathrm{~m}$.

Considering the two-way travel times to the apices of the hyperbolae, it is found that of the five transects, the minimum recorded time, as expected, was for the third one which went directly above the buried cylinder.

\section{Conclusions}

Site preparation for construction and repair works in built-up areas requires locating underground utilities in order to minimize interruption and disruptions. Such utilities may include underground pipes and cables. This study was conducted to evaluate the response of underground utility mains to ground penetrating radar. In this preliminary study, a controlled environment consisting of dry sand containing an LPG cylinder was arranged. The study demonstrates the value of geophysics in civil and environmental investigations. The procedure located the depth of burial and the sandground interface very accurately because the medium (the dry sand) had a substantially uniform permittivity.

\section{References}

[1] Daniel, D.J., Ground Penetrating Radar. IEE Radar, Sonar and Navigation Series 15. 2ND Edition, The Institution of Electrical Engineers: London, UK. 2004

[2] Luigia Nuzzo, Giovanni Leucci, Sergio Negri, Maria Teresa Carrozzo and Tatiana Quarta Application of 3D visualization techniques in the analysis of GPR data for archaeology, ANNALS OF GEOPHYSICS, VOL. 45, N. 2, April 2002, Page 321

[3] Reynolds, J.M., An introduction to applied and environmental geophysics. John Wiley and Sons. (1997) $706 \mathrm{pp}$

[4] Neal A. Ground-penetrating radar and its use in sedimentology: principles, problems and progress. - Earth-Science Reviews (2004) 66:261330.

[5] Chu, C. C., Li, Y. M., Teng, H. C and Cherng, S., The Permittivity Frequency-dependent Response of a Ceramic Material to Electromagnetic Waves at $2 \mathrm{GHz}$ Journal of American Science, (2006) 2(4).

[6] Davis, J.L., Annan, A.P., Ground penetrating radar for high resolution mapping of soils and rock stratigraphy: Geophysical Prospecting. (1989) Vol. 37.531p

[7] MALA Geoscience AB Sweden. MALA Geosciences Application note. Printed matter No. (2010) 2898. 2-3 pp.

[8] Bano, M., Loeffler, O., Girard, J.F., Ground penetrating radar imaging and time-domain modeling of the infiltration of diesel fuel in a sandbox experiment. Geosciences, (1990) 341-848

[9] Sandmeier, J. K. REFLEXW, (2008)Version 5.0.

[10] Milsom John. Field Geophysics. 3rd Edition. John Wiley. (2003)173pp. 